\title{
Overview of Car Rollover System Development
}

\author{
Songfei Zhang a, Shaoyi Bei ${ }^{\text {b, }}{ }^{*}$, Bo Li, Yingjie Jin, Hao Yan \\ Jiangsu University of Technology Mechanical Engineering Institute, China. \\ a zhangsongfei@126.com, *,b bsy1968@126.com
}

Keywords: Rollover model; rollover warning; rollover simulation.

\begin{abstract}
With the rapid development of automobile industry, automobile safety performance has become the first requirement that more and more people consider. Car rollover is a high-risk traffic accident. According to the statistics of the U.S. National Transportation Safety Administration, although vehicle rollover only accounts for $8 \%$ of traffic accidents, serious injuries and deaths are as high as $21 \%-31 \%$. China's automobile rollover research started late. The data also show that $90 \%$ of traffic accidents can be avoided if the driver is effectively alerted $1 \mathrm{~s}$ before a potential traffic accident occurs. This article summarizes the current mainstream models of rollovers, rollover warning hardware and algorithms, and calculates rollover angles through simulation. And compared the pros and cons of this kind of scheme, made a summary of the analysis and warning of rollover stability.
\end{abstract}

\section{Introduction}

Vehicle rollover is a major traffic safety accident that causes loss of life and property. Due to the large mass and structural size of heavy vehicles, the high center of mass of heavy vehicles is more likely to cause rollover accidents when the vehicle encounters extreme conditions such as small radius turns and avoiding obstacles. In order to reduce rollover accidents of heavy vehicles and improve the handling and roll stability of heavy vehicles, the necessary active safety measures must be taken to avoid the rollover of vehicles [6].

In general terms, rollover is usually divided into two categories: tripping rollover and non-tripping rollover.

Trip rollover refers to the vehicle in the process of generating a skid, when the side slipped out of the normal road, one side of the tire into the muddy road, slippery road or the body hit a similar strapless or railings Obstacles, large stumbling forces, are generated on the tire or body to cause the vehicle to rollover. When a tripped rollover occurs, the vehicle is no longer a dynamic model that is governed by the structure and parameters of the vehicle, but rather a simple rigid body motion [1], with tripped side-inverted rollovers in all rollover accidents Possession a large proportion, about $80 \%$. Another type of non-trip type rollover accounted for a smaller proportion, only about $20 \%$.

Non-trip type rollover, usually at a higher speed to avoid obstacles caused, also known as curvilinear motion rollover. Refers to the driver's lack of concentration, high speed in the process of encountering unexpected situations, the need to urgently avoid pedestrians or obstacles hurled the steering wheel so that the lateral acceleration is too large, one side of the wheel vertical reaction force is zero the resulting vehicle rollover. Non-tripped rollover by the vehicle's own parameters, road input, driving conditions and external factors such as the comprehensive interference decision is the main research vehicle rollover. Traditional rollover evaluation using simple roll angle or lateral acceleration [16], the method of real-time and accuracy is poor; relative to the traditional rollover evaluation criteria, based on estimates of tire deformation to determine Rollover [17] and rolloverbased algorithm (TTR) [18] It is important to note that non-tripped rollover, although a small percentage of all rollovers, generally occurs at the center of gravity Tall, bulky heavy vehicles. If it happens, it will bring more loss $[7,10]$.

The working process of vehicle rollover control system is generally divided into two phases: the first phase is to estimate the possibility of vehicle rollover, which is mainly through measuring the vehicle's driving parameters (wheel lateral load transfer rate, rollover angle, Folding angle, lateral acceleration, yaw rate and folding angular velocity, etc.) to estimate the possibility of rollover, so as to decide whether to open the rollover prevention control system. The second stage is when the 
estimation result exceeds the preset side turn the door limit, turn on the rollover control system, by changing the vehicle's driving status to prevent the vehicle rollover. Therefore, the core technology of vehicle rollover control is essentially to estimate the possibility of vehicle rollover and to reduce the possibility by rollover control system to ensure the safe driving of vehicles [9].

\section{The Establishment of Car Rollover Model}

The model assumes the following assumptions: The car is steered so it does not take into account the effects of longitudinal motion; ignores the effects of air forces; neglects unsprung mass effects, ie, does not account for tire mass and vertical deformation; Characteristics of the impact of rollover; suspension simplified to the ideal torsion spring with damping; the same time, assuming that the lateral speed and yaw rate relative speed is small, linearization can be processed. I think one of them. The most basic is the establishment of three-degree-of-freedom vehicle rollover model [2].

\subsection{Three Degrees of Freedom Model}

I refer to Jilin University, Li Zhanqi three-degree-of-freedom car model to explain in detail, to briefly introduce the establishment of three-degree-of-freedom car model.

A three-DOF vehicle model adds a degree of roll freedom based on a two-degree-of-freedom vehicle model, along with a total of three direction-finding movements along the $Y$-axis, a yaw around the Z-axis, and a roll around the $\mathrm{X}$-axis The degree of freedom model uses the previous wheel angle as input. Make the following assumptions before establishing the vehicle dynamics equations:

Do not consider the role of aerodynamics.

Do not consider the change of the tire characteristics caused by the lateral movement of the left and right wheels due to the load.

The car travels on a level road, ignoring the vertical movement of the car along the $\mathrm{Z}$ axis and the pitch movement around the $\mathrm{Y}$ axis.
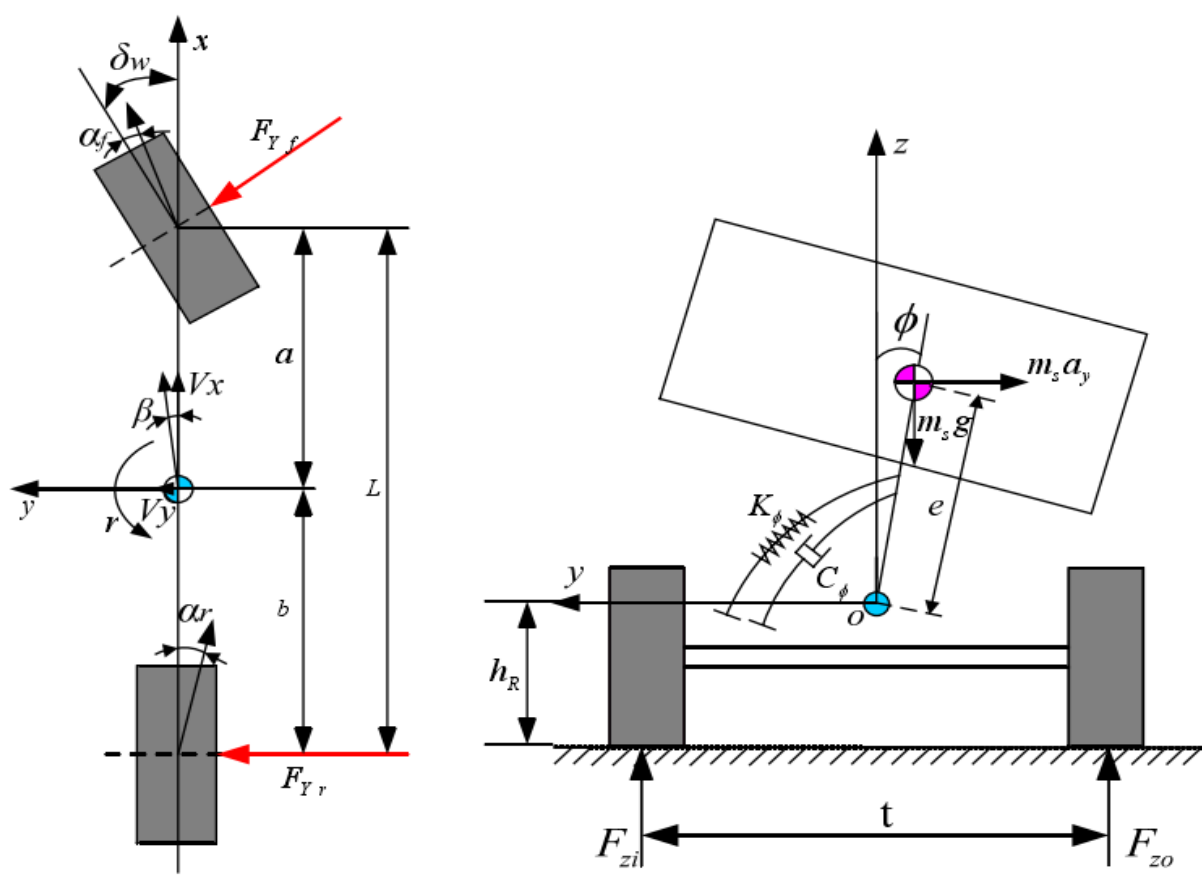

Figure 1. Three-Degree-of-Freedom Car Model Diagram

According to the assumptions, the equilibrium equations for the $\mathrm{Y}$ direction, around the $\mathrm{Z}$ axis and around the $\mathrm{X}$ axis are listed according to the Darling principle:

$$
\begin{gathered}
\sum F_{y}=m \cdot\left(r \cdot V_{x}+\dot{V}_{y}\right)-m_{s} \cdot e \cdot \ddot{\Phi} \\
\sum M_{s}=I_{s} \cdot \dot{r}+I_{x S} \cdot \ddot{\Phi} \\
\sum M_{x}=I_{x s} \cdot \ddot{\Phi}-m_{s} \cdot e \cdot\left(r \cdot V_{x}+\dot{V}_{y}\right)+I_{x s s} \cdot \dot{r}
\end{gathered}
$$

The three together as follows:$$
\sum F_{y}=F_{Y f}+F_{y r}=K_{f} \cdot \alpha_{f}+K_{r} \cdot \alpha_{r}
$$ 


$$
\begin{gathered}
\sum M_{s}=a \cdot F_{Y f}+b \cdot F_{Y r}=a \cdot K_{f} \cdot \alpha_{f}-b \cdot K_{r} \cdot \alpha_{r} \\
\sum M_{x}=m_{s} \cdot g \cdot e \cdot \phi-K_{\phi} \cdot \phi-C_{\phi} \cdot \dot{\phi}
\end{gathered}
$$

We combine the above formula, and the other $\mathrm{P}=\dot{\varphi}$, can be obtained three-degree-of-freedom vehicle model motion differential equation matrix is as follows:

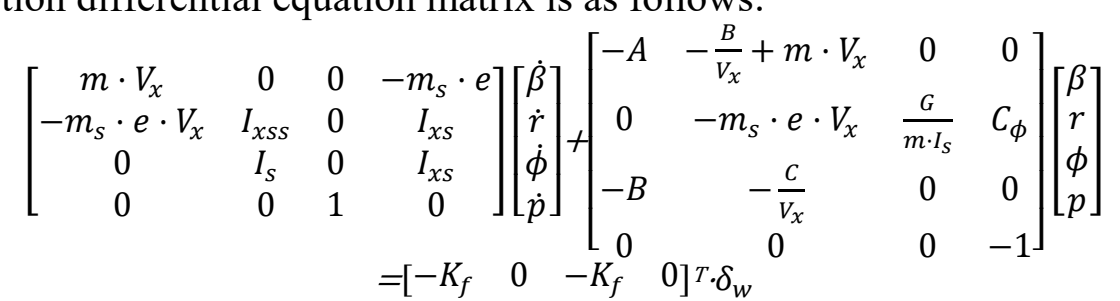

In the above formula, $X=[\beta, r, \varphi, p]$ is the state equation of state:

Where:

$$
\left\{\begin{array}{c}
\dot{\beta}=\frac{1}{m \cdot V_{x}}\left[\begin{array}{c}
\left(\frac{m_{s} \cdot e \cdot E}{D}+A\right) \cdot \beta+\left(\frac{m_{s} \cdot e \cdot F}{D V_{x}}+\frac{B}{V_{x}}-m \cdot V_{x}\right) \cdot r-\frac{m_{s} \cdot e \cdot G}{D} \cdot \phi \\
-\frac{m_{s} \cdot e \cdot H}{D} \cdot p+\left(\frac{m_{s} \cdot e \cdot J}{D}-k_{f}\right) \cdot \delta_{w}
\end{array}\right] \\
\dot{r}=\frac{1}{I_{z}}\left[\left(\frac{-I_{x s} \cdot E}{D}+B\right) \cdot \beta+\left(\frac{-I_{x s} \cdot F}{D \cdot V_{x}}+\frac{C}{V_{x}}\right) \cdot r+\frac{I_{x s} \cdot G}{D} \cdot \phi+\frac{I_{x s} \cdot H}{D} \cdot p\right] \\
-\left(\frac{I_{x s} \cdot J}{D}+k_{f} \cdot a\right) \cdot \delta_{w} \\
\phi \doteq p
\end{array}\right]
$$

$$
\begin{gathered}
D=-m_{s}^{2} \cdot e^{2} \cdot I_{s}-m \cdot I_{x S} \cdot I_{x S S}+m \cdot I_{s} \cdot I_{x S} \\
E=m_{s} \cdot e \cdot A \cdot I_{s}-m \cdot B \cdot I_{x s s} \\
F=m_{s} \cdot e \cdot B \cdot I_{s}-m \cdot C \cdot I_{x s s} \\
G=m \cdot I_{s} \cdot\left(K_{\phi}-m_{s} \cdot g \cdot e\right) \\
H=m \cdot I_{s} \cdot C_{\phi} \\
J=m \cdot a \cdot k_{f} \cdot I_{x s s}-m_{s} \cdot e \cdot k_{f} \cdot I_{s}
\end{gathered}
$$

In the above formulas, $\mathrm{m}_{\mathrm{s}}$ is the sprung mass of the vehicle, $\varphi$ is the roll angle of the vehicle body, $\mathrm{p}$ is the roll angle of the vehicle body, $\mathrm{K}_{\phi}$ is the roll stiffness of the vehicle, $\mathrm{C}_{\phi}$ is the roll damping of the vehicle, Ixs is the vehicle sprung mass around the vehicle $\mathrm{X}$ axis inertia of the coordinate system, $\mathrm{I}_{\mathrm{xs}}$ is the inertial product of the vehicle mass about the $\mathrm{X}, \mathrm{Z}$ axis of the vehicle coordinate system, $\mathrm{I}_{\mathrm{xss}}$ is the inertial product of the vehicle sprung mass around the $\mathrm{X}, \mathrm{Z}$ axis of the vehicle coordinate system, and e is the centroid of the vehicle sprung mass The distance to the roll center.

The above is a most basic rollover model, when the car rollover occurs, the lateral acceleration of the car will reach a larger value, and the deformation of the tire under rollover will also be in a nonlinear state. Therefore, the pavement adhesion limit needs to be taken into account for the tire Lateral force limitations. In actual operation of the sub-processing is a commonly used method of processing. Correspondingly, the $\mathrm{K}_{\mathrm{f}}$ and $\mathrm{K}_{\mathrm{r}}$ in the equation of state of a vehicle with three degrees of freedom must be segmented accordingly. Only in this way can we construct a more adaptable non-linear degree of freedom vehicle model.

Of course, there are many models to establish the model, the establishment of the model of three degrees of freedom is the most basic. In the reading of the literature, I found that Huang Mingliang's paper in Hunan University mentioned that the establishment of 10-DOF model is more accurate. The main idea of establishing the 10-DOF model of Huang Mingliang is that the sprung mass is a freewheeling point The mass of rotation, mass $\mathrm{m}_{\mathrm{s}}$, inertial tensor Is; The unsprung masses are four rigid bodies that move vertically only in the vehicle coordinate system, mass $m_{u i}(i=1,2,3,4)$. The ten degrees of freedom are: three directions of sprung mass translation and three axial rotation, four vertical unsprung mass beating [3].

The above is a preliminary understanding and summary of the automobile modeling when I read the articles by Jin Zhilin, Li Zhanqi, Huang Mingliang and others. Of course, this model there are many deficiencies, this model can only reflect the moment when the car rollover situation, for the prevention of rollover and did not play a good preventive effect. Therefore, a more specific and 
comprehensive model needs to be established to serve as a long-term model that can be predicted before the rollover occurs.

\subsection{Drum Model}

When researching vehicles for tumbling motion, especially for car tumble accidents, the vehicle can be simplified into a roller model. Drum model is an effective model that can be used in the actual car rollover accident reproduction. It has the advantages of less parameters and less calculation. This model approximates the vehicle to a roller of the same mass $\mathrm{m}$, the same axis of rotation ( $\mathrm{x}$-axis over center of mass) as the roll-over vehicle, with rollers of the same moment of inertia $\mathrm{I}_{x x}$ rolling on the road surface. Roller model and the ground effective friction factor $f$, as shown.
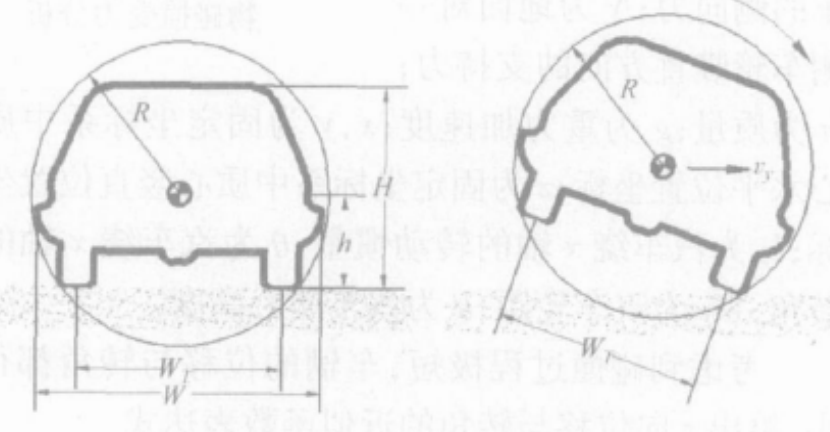

Figure 2. Tumbling Roller Model

Roll model rollover expression:

$$
\left\{\begin{array}{c}
\varepsilon=\operatorname{sign}\left(V_{y}-\omega R\right) m f R / I_{x x} \\
\omega=\int \varepsilon d t \\
\theta=\int \omega d t=\iint \varepsilon d t
\end{array}\right.
$$

Where $\varepsilon$ is the angular acceleration; $\omega$ is the angular velocity; $\mathrm{R}$ is the radius of the cylinder; $V_{y}$ is the lateral translational velocity of the centroid [1].

\section{Common Rollover Warning Principle}

\subsection{Basic Rollover Warning Principle}

Early rollover warning systems rollover warning of vehicles by establishing static thresholds (eg lateral acceleration, roll angle threshold, etc.). With the development of rollover early warning research, the emergence of the early warning algorithm using dynamic stability threshold has the advantages of good accuracy. Which appeared earlier there are several:

LTR: The concept of using the lateral load transfer to judge the vehicle rollover proposed by Rakheja S. The lateral load transfer is estimated by the ratio of the leaf spring force on both sides. LTR is defined as the ratio of the difference between the vertical load and the sum of the vertical loads on the wheels on both sides of the vehicle $\left(L T R=\frac{\left|\sum_{i=1}^{n}\left(F_{l i}-F_{r i}\right)\right|}{\sum_{i=1}^{n}\left(F_{l i}+F_{r i}\right)}\right)$ Where $\mathrm{F}_{\mathrm{li}}$ is the vertical load on the left wheel of the vehicle; $F_{r i}$ is the vertical load on the right wheel of the vehicle; $i$ and $n$ are the position of the shaft and the total number of axles, respectively. When the vehicle is rolling, the vertical loads are redistributed on the left and right wheels. The range of the LTR is $[-1,1]$. When $\mathrm{LTR}=0$, the car will not roll and will not rollover. When the LTR $= \pm 1$, one side of the vertical load is 0 , the tire off the ground, the risk of rollover. In more literature, the limit of LTR is 0.9 [8]. The LTR and its deformation can intuitively reflect the real-time rolling state of the vehicle, so it is widely used in the industry [19-21].

RPER: The vehicle from the current position side flip side position, the required energy difference. During the rollover process, the energy and gravitational potential energy are transformed into each other. The rollover condition RPER value is less than 0 ; non-rollover condition RPER value is greater than 0 . 
RSF: Definition and LTR difference of an absolute value. The value of RSF is between -1 and 1. $\left(R S F=\frac{\sum_{i=1}^{n}\left(F_{r i}-F_{l i}\right)}{\sum_{i=1}^{n}\left(F_{r i}-F_{l i}\right)}\right.$

TTR: Reality is far more complicated than theory, so choose a good car rollover theory become the key point.

\subsection{Rollover Research Energy Method}

By reading Huang Mingliang Hunan University thesis found that the applicability of energy law is very wide, here I briefly introduce the idea.

Rollover and energy: consider only the lateral kinematics of the vehicle dynamics model can be simplified as shown in the vibration system: the base is divided into two equal parts of the massm $_{u}$; Sprung part can rotate around the $\mathrm{O}$ point, $\mathrm{O}$ point can be moved up and down in the vertical slide rail, sprung mass $m_{s}$, around the $O$ point moment of inertia $I_{s}$, centroid to the $O$ point distance $h_{s}$; spring mass excluding stiffness $\mathrm{k}_{\mathrm{s}}$; damper quality, Damping size is $\mathrm{C}_{\mathrm{s}}$.

$$
\left[\begin{array}{cc}
m_{s} & m_{s} h_{s} \sin \varphi \\
m_{s} h_{s} \sin \varphi & m_{s} h_{s}^{2}+I_{s}
\end{array}\right]\left\{\begin{array}{c}
\ddot{Z_{s}} \\
\ddot{\varphi}
\end{array}\right\}+\left[\begin{array}{cc}
2 C_{s} & 0 \\
0 & 2 C_{s} t_{e}^{2}
\end{array}\right]\left\{\begin{array}{c}
\ddot{Z}_{s} \\
\dot{\varphi}
\end{array}\right\}+\left[\begin{array}{cc}
2 k_{s} & 0 \\
0 & 2 k_{s} t_{e}^{2}
\end{array}\right]\left\{\begin{array}{c}
Z_{s} \\
\varphi
\end{array}\right\}=\left\{\begin{array}{c}
0 \\
m_{s} g h_{s} \sin \varphi
\end{array}\right\}
$$

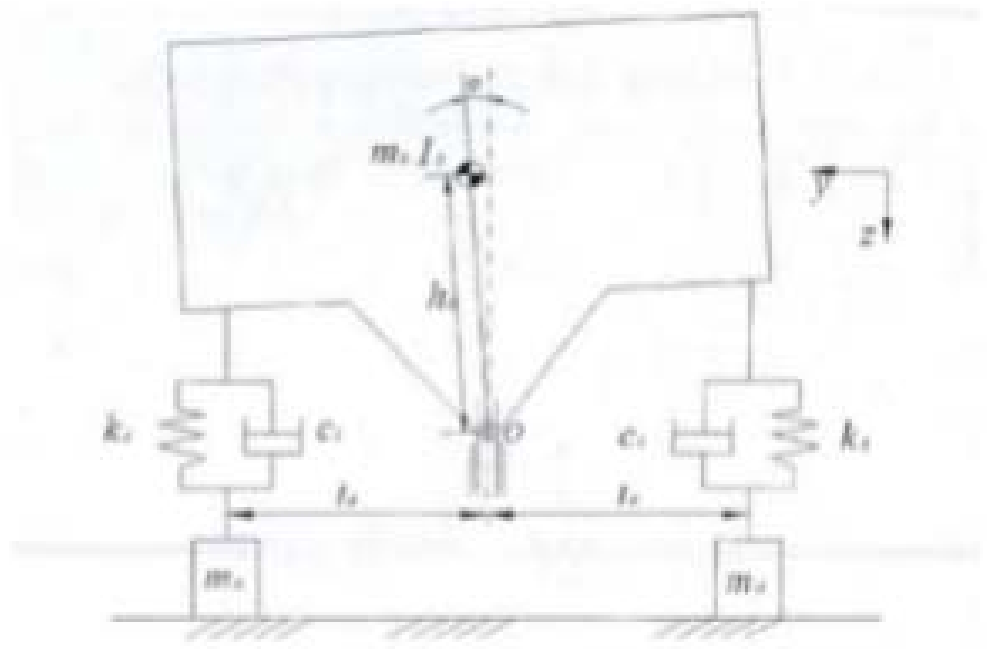

Figure 3. Car roll Motion Diagram

The greater the mechanical energy of the system, the greater the probability of unsprung mass leaving the ground. The magnitude of the mechanical energy that the system has to a certain degree determines the possibility of rollover of the vehicle. Moreover, using the system energy as the evaluation index can avoid the dependence on the interaction force between the ground and unsprung mass, and thus has universal applicability.

Definition of rollover energy threshold: The rollover energy threshold is defined as the minimum energy required for the vehicle to rollover from horizontal to rollover. The energy threshold can be determined by the vehicle (quasi-) static rollover test, for example: side-pull test, tilt test stand, etc. It can also be calculated by measuring the vehicle structural parameters. According to the principle of slowly tilting the test bench, the lifting process is quasi-static, and the change of kinetic energy can be neglected. The difference $\Delta \mathrm{E}_{\mathrm{p}}$ between the front and rear potential energy is the minimum energy required for the vehicle to pass from static to rollover. According to the existing calculation method, the maximum rollover angle of the model can be calculated. According to the maximum rollover stability angle, the height change of vehicle sprung mass and unsprung mass centroid can be calculated and $\Delta \mathrm{E}_{\mathrm{p}}$ can be obtained. When the difference of potential energy calculated by the above method is used as the threshold energy of rollover, the influence of tire deformation on unsprung mass center of mass and vehicle roll and the change of sprung mass center height due to roll deformation of suspension are neglected. In order to reduce the influence of above factors on rollover 
energy threshold, the threshold stability coefficient $\lambda_{\text {et }}$ is introduced, whose size is determined by tire stiffness and the energy threshold is defined as $\mathrm{E}_{\mathrm{t}}=\lambda_{\mathrm{et}} \Delta \mathrm{E}_{\mathrm{p}}$.

The energy method of verification: In order to verify the correctness of energy methods and stability indicators, the need to use the existing rollover indicator validation, the choice of widely used LTR factor as a comparison to verify. As the energy stability index is always positive, it is necessary to change the absolute value of LTR to

$$
|L T R|=\frac{\left|\left(F_{z 1}+F_{z 3}\right)-\left(F_{z 2}+F_{z 4}\right)\right|}{F_{z 1}+F_{z 3}+F_{z 2}+F_{z 4}}
$$

In MATLAB simulation of a variety of conditions of the vehicle operating conditions, respectively, using two indicators to judge and contrast [3].

The above is a brief description of Huang Mingliang's energy law. The method verifies the limitations of the transverse load transfer rate and the applicability of the energy method, and better reflects the wider use of energy hair.

\section{Conclusion and Prospect}

Relatively late start in the relevant research in China, but also made some achievements. He Feng and Yang Liyong [24] from Guizhou University of Technology conducted a series of researches on rollover stability of vehicles and achieved results in rollover stability simulation and stability control, especially in non-fully loaded tank cars the rollover analysis conducted in-depth study. Nanjing Forestry University, Liu Jing [25] conducted a tanker anti-rollover body attitude control and alarm strategy, and in the ADAMS simulation analysis, taking into account the center of mass changes on the rollover, but has not considered the tire side Slip on the impact of vehicle rollover. With the rapid development of transportation, the number of traffic accidents is on the rise, and major traffic accidents such as rollover are especially prominent. Nowadays people have been very concerned about the issue. In the past rollover research by scholars at home and abroad, more attention has been paid to the study of rollover detection and rollover control, and there are few studies on rollover warning. The rollover early warning can effectively reduce the rollover caused by man-made accidents, with a greater research value. The purpose of this paper is to provide a reference solution for the design and testing of vehicle stability, rollover warning and control systems. At the same time, we also understand the energy law of vehicle rollover stability dynamics research. My future research is also inspired.

\section{Acknowledgements}

This project is supported by the National Natural Science Foundation of China (Grant No. 51705220), the Jiangsu Province Higher Education Natural Science Research Project (17KJD580001) and the Jiangsu Provincial Higher Education Natural Science Research Major Project (17KJA580003) and Foundation for Jiangsu Province "333 Project" Training Funded Project (BRA2015365), Changzhou Science and Technology Support Project (Industry) (CE20150084).

This project is supported by the National Natural Science Foundation of China (Grant No. 51705220), the Jiangsu Province Higher Education Natural Science Research Project (17KJD580001) and the Jiangsu Provincial Higher Education Natural Science Research Major Project (17KJA580003) and Foundation for Jiangsu Province "333 Project" Training Funded Project (BRA2015365).

\section{References}

[1]. Zhu Jun, Li Yibing. Vehicle rollover and rollover accident modelling [J]. Automotive Engineering, 2006 (03): 254-258.

[2]. Jin Zhilin, WENG Jiansheng, HU Haiyan. Vehicle rollover warning and rollover control [J]. Chinese Journal of Dynamics and Control, 2007 (04): 365-369. 
[3]. Huang Mingliang, Zheng Minyi, Zhang Bangji, Zhang Nong, Chen Shengzhao. Study on vehicle rollover stability dynamics based on energy method [J]. Journal of Vibration and Shock, 2016, 35 (24): 164-174.

[4]. Li Zhanqi. Dynamic control of vehicle yawing and rollover stability based on differential braking [D]. Jilin University, 2011.

[5]. Huang Jieyan, Liu Kun, Xiong Yi. Research on the status of vehicle rollover warning and control system [J] .Large Light Vehicle Technology, 2011 (Z1): 16-19 + 23.

[6]. Xia Jingjing, Chang Lv, Hu Xiaoming, HE Yong. Rollover Active Control of Heavy Vehicles Based on Model Prediction [J]. Journal of Agricultural Engineering, 2010, 26 (09): 176-180.

[7]. Li Junjie. Vehicle rollover warning system dynamics simulation and algorithm [D]. Wuhan University of Technology, 2013.

[8]. Zenda Xi, Hu Shugen, Wang Yun, Song Xiaowen. Study on anti-rollover dynamics of automobile differential brake based on LTR [J]. Mechatronics, 2011, 28 (05): 532-536 + 541.

[9]. YE Shu-jian. Study on TruckSim-based Anti-Rollover Control for Heavy-Duty Trains [D]. Guangxi University, 2013.

[10]. Zhu Tianjun. Research on rollover early warning and multi-objective stability control based on improved TTR heavy vehicle [D]. Jilin University, 2010.

[11]. Song Xiaowen. An improved vehicle rollover model and its application [A]. China Society of Automotive Engineers .2009 China Society of Automotive Engineers [C]. China Society of Automotive Engineers: 2009: 1.

[12]. Qin Hongwu, Shi Cunjie, Liu Jun, Wei Longping, Yu Festival hair. Intelligent vehicle sensing and rollover early warning system design [J]. Sensors and Microsystems, 2012.

[13]. Journal of Sensors and Microsystems, 2011, 30 (11): 83-85. Zhang Tianheng, Peng Donglin, Yang Jisen, Zheng Fangyan. Dynamic measurement and data processing of linear time-grid sensor $[\mathrm{J}]$.

[14]. Orlov D A, Neverova E A, Dmitriev S V. Turning angle sensorbased on a periodic optical raster and photodetector matrix in full-frame mode [J]. Measurement Techniques,2011,54(9):1065-1069.

[15]. Ou Jian, Cheng Xiangchuan, Zhou Xinhua, Yang Echuan, Zhang Yong. Rollover control strategy based on vehicle stability control system [J]. Journal of SouthWest Jiao Tong University, 2014, 49 (02): 283-290.

[16]. RAKHJA S, PICHE A. Development of directional stability criteria for an early warning safety device[R]. SAE Paper, 1990:902265.

[17]. TRENT V, GREENE M. A genetic algorithm predictor for vehicle rollover [R]. IECON02, IEEE 28th Annual Conference, 2002.

[18]. Chen B, Peng H. Differential-braking-based rollover prevention for sport utility vehicles with human-in-the-loop evaluations [J]. Vehicle System Dynamics, 2001, 36 (4/5): 359 -389.

[19]. Zuo Shuguang, Zhang Haopeng, Zhao Yuanling, Wu Xudong, Ni Tianxin. Study on the selfexcited vibration of driving wheel and driven tire tread [J]. Journal of Vibration and Shock, 2014, 33 (19): 17-21.

[20]. CHENBC, PENGH. Rollover warning for articulated heavy vehicles based on a timetorollover metric [J]. ASME Journal of Dynamic Systems, Measurement and Control, 2005, 127(3), 406-414. 
[21]. CHOISB. Practical vehicle rollover avoidance control using energy method [J]. Vehicle System Dynamics, 2008, 46(4):323-337.

[22]. Zhang not Yang, Zong Chang-fu, Wang Deling. Vehicle rollover early warning research status and key technologies [J]. Automotive Technology, 2010 (08): 27-31.

[23]. Huang Jie Yan. TTR early warning based on heavy vehicle rollover control system [D]. Jilin University, 2008.

[24]. Cheng Guanghua. TTR-based vehicle rollover early warning device design [D]. Nanjing University of Aeronautics and Astronautics, 2008.

[25]. Liu Jing. Tanker anti-rollover posture control and alarm strategy simulation analysis [D]. Nanjing Forestry University, 2009. 